\title{
Mobile Robot for Automatic Installation of Floor Tiles
}

\author{
Dimitrios Apostolopoulos 1 \\ da1v@cmu.edu
}

\author{
Hagen Schempf ${ }^{1}$ \\ hagen+@cmu.edu
}

\author{
Jay West ${ }^{2}$ \\ jwest@isscad.com
}

\author{
1. The Robotics Institute \\ Carnegie Mellon University \\ Pittsburgh, PA 15213
}

\begin{abstract}
This paper describes the configuration of a floor-tile installation robot for commercial buildings. The research is motivated by the need to reduce the installation time and cost while guaranteeing consistent quality. In order to compete with human installation, a time of 24 seconds per installed tile has to be matched. The technical solution that is deemed feasible and capable of reducing this time to about 10 seconds, is an autonomous, electrically-powered mobile robot with omni-directional locomotive capability, and stereo cameras and light-striper for sensing. High resolution imaging is needed to identify tile seams and edges, assess the quality of automatic installation, and locate where the next tile should be placed. A mechanically compliant placement device would place the tile quickly and accurately without damaging the placed and surrounding tiles, emulating a human capability. Vinyl/ceramic tiles, adhesives, and grout are carried onboard the robot and replenished by the operator. Navigation and positioning are performed through a laserbased triangulation system, and by detecting, counting and dead-reckoning off of tiles placed on the floor. Tile and installation quality are continuously monitored and errors corrected for, based on an overall layout map.
\end{abstract}

\section{Introduction}

Tile installation on large floor areas is a task that has to be performed efficiently, accurately, and repeatedly. It requires optical inspection and tactile/force sensing in very large areas, such as a 100,000 square foot supermarket. Optical inspection and tile placement phases are monotonous tasks for humans and can be performed efficiently only for limited time and at unreliable levels of intensity. The installer has to avoid erroneous seam openings and runouts, keep track of the points where the errors do appear and also understand how to correct for them.

An autonomous robot with appropriate mobility and perceptual capabilities is a promising solution to automating the tile installation task for the purposes of improving quality, while reducing time and dependency on a scarce skilled labor force. Such robot has be able to handle, feed and place the tiles with very low accuracy.
2. Integrated Silicon Systems, Inc. Research Triangle Park, NC 27709
In this paper we profile the tile installation task and present a solution to the perception problem of assessing tile-placement quality. We describe a robot configuration suitable for automatic tiling, and establish metrics for comparing robot to human performance.

\subsection{Floor-Tile Installation}

The current installation process is carried out by skilled human installers. The phases of the installation process which will have to be closely replicated by the robotic installation device [1], [2], as well as those phases which can and need to be modified to allow robotic automation, are to:

- Center and square off the room.

- Apply the proper adhesive; it usually takes 3 to 4 days. for the adhesive to fully set (the way the adhesive is spread may have the largest influence on the quality of the installation).

- Install the tiles in a step or fan pattern propagating outwards from the central grid layout.

- Scribe pattern or/and fit tiles in order to complete the installation (fitting irregular walls or door trims).

- Roll the floor after tile installation is complete.

A typical installation would be a 12" x 12" Imperial Tile in a 40,000 $\mathrm{ft}^{2}$ supermarket; a mechanic and a helper would be expected to place 2,500 to $3,000 \mathrm{ft}^{2}$ per day at an hourly rate of $\$ 26.00$, which means that it takes $10-12$ seconds to install a tile (or 312-375 tiles/hour) and it costs about $\$ 2,800$ - $\$ 3,400$ to install the $40,000 \mathrm{ft}^{2}{ }^{2}$ floor. [3]

Tile Attributes: The variety of sizes and shapes of tiles gives an indication of the handling and placement problem that this robot system will have to address. Furthermore basic robot size and subcomponent complexity can be initially inferred from the need to package and handle a variety of different tile shapes and sizes in order to lay a completely arbitrary pattern. [1] [2] [3]

Patterns: An important issue of any installation job, is the variety of tile patterns that can be created from the basic product line. The variety of possible patterns for the various types of tiles has a dramatic effect on the sensing modes and techniques suggested and analyzed later in this 
paper. The conclusion from this data, is that some form of visual sensing mode that is insensitive to or knowledgeable about possible patterns will be needed to perform any kind of visual assessment or positioning tasks for the mobile robot.

\section{Tile Placement Quality}

Critical to automating installation of floor tiles is the issue of real-time evaluation of tile placement quality. In order to establish the proper metrics and the respective numeric bounding parameters that define tiling quality we have classified tiling patterns, identified possible placement errors, and measured tile placement parameters that affect human judgement of tiling quality. We worked on two methods for evaluating the quality of the installation: Assessment based on measurements of: (1) tile corner intersections and (2) tile seams. In the first method the measurements are taken at intersections of tile corners, whereas in the second method the measurements are taken along all the seams of each adjoining tile. Both methods require a camera-based vision system to obtain the images, yet relative processing complexity and achievable data reliability, density, and accuracy favored the former of the two approaches: Tiling quality assessment based on tilecorner intersections.

\subsection{Classification of Installation Errors}

One of the most important criteria in tiling quality assessment is to define the types of defects that are expected when processing an image. The following types of defects have been classified as being a necessary and sufficient set of descriptors for any 4-tile corner. Compound defects, such as shift in two dimensions, are combinations of the basic defects:

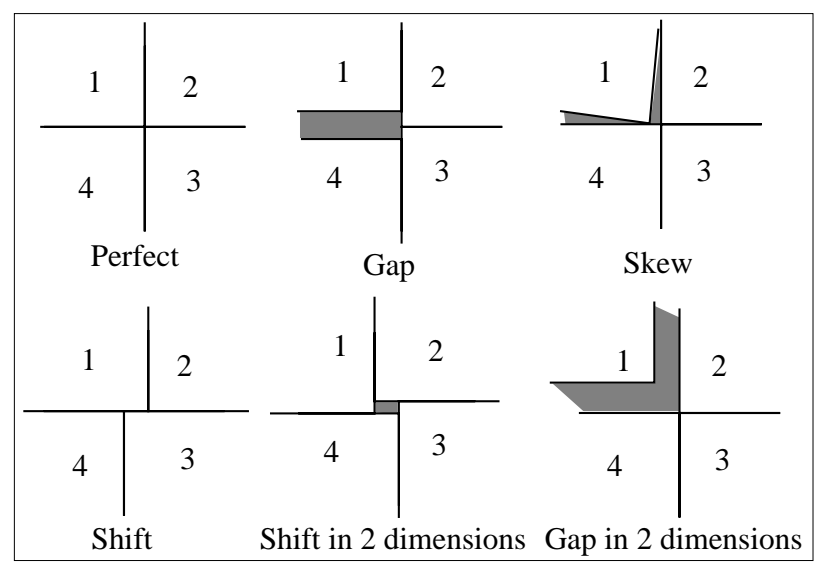

Figure 1: Tiling installation defects

For each intersection that is analyzed, 4 sets (since there are 4 corners) of 3 parameters (skew, gap, shift) can be generated to characterize each intersection. Thus if we analyze 4 different tile corners, we will generate 12 differ- ent parameters, yet in order to describe all the degrees of freedom for all corners, we only need 9 parameters namely the position and orientation of each tile corner w.r.t. one corner labelled as the reference frame. It is obvious that we have redundant parameters, which can only be explained through dependency relations between the four tiles in question. In a final implementation, the redundancy could certainly be resolved by limiting the data set to only those parameters that are independent.

The independent measurements can thus be performed using the representation shown in Figure 2 for each of the different defects that were identified:

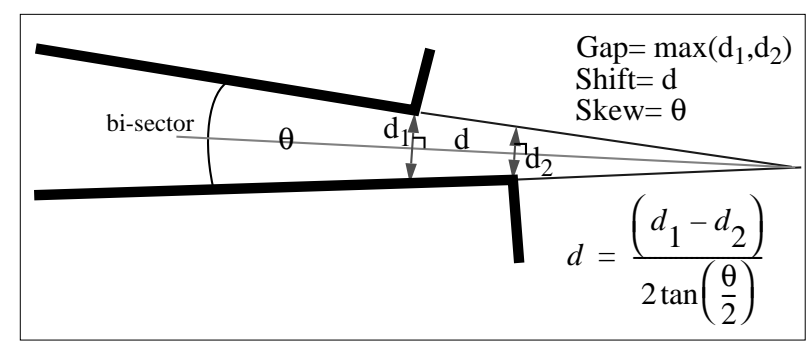

Figure 2: Tiling defects definitions

The conventions illustrated in Figure 2 can thus be used to yield important tiling quality descriptors. These descriptors were applied to the image gathered from ceramic tiles (without grout), where the video image is shown in Figure 3. The results that were obtained for each of the four tiles at the intersection are tabulated below:

\begin{tabular}{|l|c|c|c|c|}
\hline \multicolumn{1}{|c|}{ Flaw } & tile 1 & tile 2 & tile 3 & tile 4 \\
\hline \hline Gap & $0.14 "$ & $0.32^{\prime \prime}$ & $0.18^{\prime \prime}$ & $0.29 "$ \\
\hline Skew & $-0.18 \mathrm{deg}$ & $-0.18 \mathrm{deg}$ & $\sim 0 \mathrm{deg}$ & $\sim 0 \mathrm{deg}$ \\
\hline Shift & $0.032^{\prime \prime}$ & $0.047^{\prime \prime}$ & $-0.032^{\prime \prime}$ & $0.039^{\prime \prime}$ \\
\hline
\end{tabular}

Table 1: Measurements of tile defects

\subsection{Characterization of Tile Placement Quality}

In order to explore the perceptual issues associated with camera images, images of vinyl and ceramic tiles were taken. The tiles represented a cross section of reflective properties expected to be encountered, from highly specular to highly diffuse. Two well known low level image processing techniques, the Canny operator and the Hough transform, were applied to the images [4], [5]. The Canny operator detects the edges in the tile images and the Hough transform is used to model the strongest lines in the edge image. The overall processing time was about 6 seconds on a Sun SparcStation 1+, and the results of these processing techniques are shown in Figure 3. 


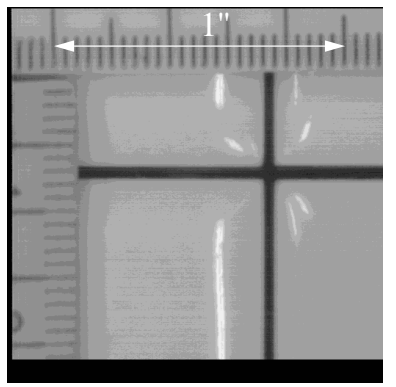

(a)

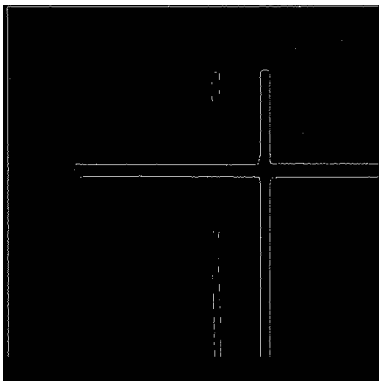

(b)

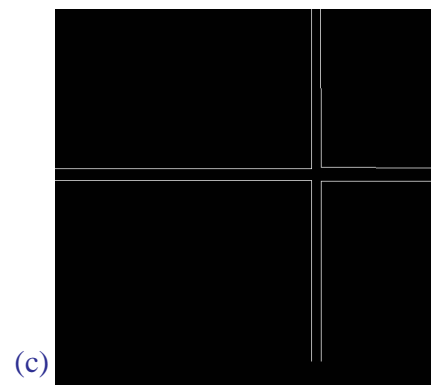

Figure 3: (a) video image of four-tile intersection, (b) edge image from Canny operator, (c) Hough transform

The resolution of the camera used in these experiments was about 16 pixels per mm. Due to the resolution of the cameras themselves, where over a $3 \mathrm{~cm}$ square field of view a 460 x 460 square CCD array camera could obtain a resolution of $0.065 \mathrm{~mm} / \mathrm{pixel}$, accurate tile seam imaging would certainly be possible.

The edge image in Figure $3 \mathrm{c}$ is relatively noise free, with distinctly visible edge points outlining the tile, which represents the best case results. Such results can be expected for ceramic tiles and for vinyl tiles which have a clear seam-like pattern already stamped/printed onto them. If we choose to image tiles which we know will cause the vision system to have problems we will get images like the ones shown in Figure 4. The video image shows a very patterned tile which has accentuated seams printed onto it, and which has a rough surface which will cause substantial specular reflection. The abundance of edges found through the Canny-operator are shown in Figure $4 \mathrm{~b}$.

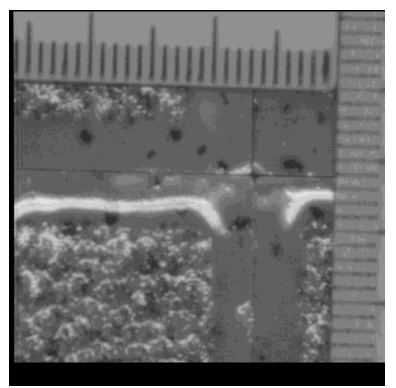

(a)

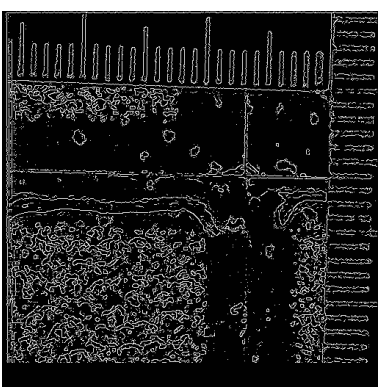

(b)
Figure 4: (a) video image of highly reflective tiles, (b) edge image from Canny operator
These worst case results illustrate the potential problems with camera image processing. First, the reflective properties of the tile itself may be inhospitable to simple image processing techniques. In the above case, the tile surface was rough and had a glossy coating, producing substantial specular reflections in all directions. Second, ignoring the specular noise, the tile boundary itself may not be perceptible to the camera. This occurs when the tiles are packed so tightly together that the tile edges seem to merge together.

The second sensor that was evaluated was a light stripe sensor. In contrast to the camera images, processing of the light stripe data was extremely simple, involving a simple threshold operation. The computer-representation of the sampled data is shown in Figure 5.

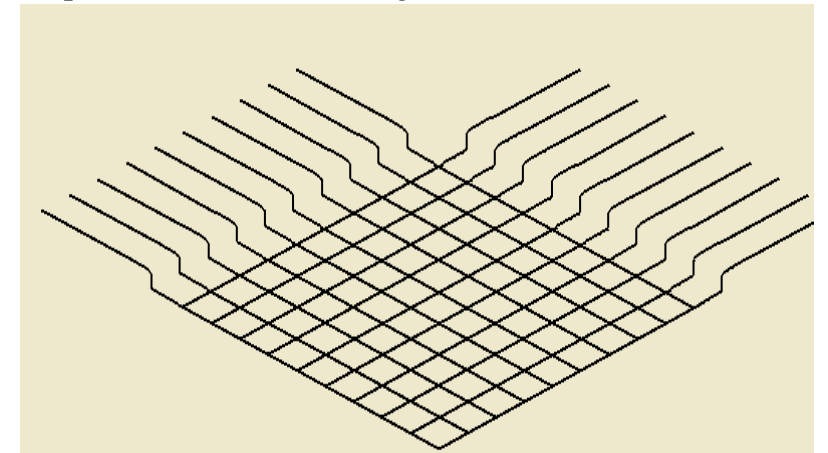

Figure 5: Composite light-stripe image of two-tile and floor intersection

Note how clear and crisp the image is, and how clear the transition between tile-to-floor and tile-edge to tile-edge is. Such data can be processed to determine the tile edge and the intersection of two mutually adjacent tile edges. Such information, coupled to the spatial location and orientation of these tile edges, will provide sufficient information to facilitate the placement of the next tile into the void defined by the two-tile corner. Further experimentation is required to determine the exact number of lightstriper-sensors and their preferred configuration to achieve sufficiently accurate edge location and orientation measurements. Building and/or acquiring miniature lightstripe sensors without excessive calibration requirements could make this approach the most simple and effective sensing mode for tile placement.

\section{Robot Configuration}

The terrain of deployment is the indoor environment of a commercial facility with obstacles of limited size expected to be overcome only during the initial access to and the final retrieval of the robot from the facility. Normal operation will be on an extremely flat floor, where the only obstacles are facility obstacles are facility features, like columns. 
Locomotion: Possible locomotion schemes include a separate and dedicated drive/steer module with two motors and transmissions per wheel, or an omni-directional Mecanum wheel [6], [7], with a single motor/transmission arrangement per wheel. The mobility requirements for task completion and the facility constraints dictate the need for providing omni-directional motion capability [8]. Motion coordination of omni-directional wheels allows for in-place turning and fine adjustments of vehicle heading, important attributes to accurately position the robot.

Tile Hopper and Feeder: In order to provide for a large number of tiles on-board the vehicle, thus reducing reloading frequency, storage space for tiles should be provided onboard the robot. Different types of tiles can be loaded in hoppers in order to generate certain patterns. The challenge thus lies in assembling a simple and intelligent tile pick-place-and-feed mechanism that picks a tile from the correct hopper, places it on a conveyor system, which in turn feeds it to a tile placement system. A friction-feed belt device would work, as it can deal with various tile sizes, weights and thicknesses. The tile pick-up device is designed to be a simple translating and pivoting frame with suction cups to lift and rotate the tile onto the beltmechanism.

Fine Positioning Device for Tile-Vision and Placement Systems: Several mechanical manifestations using springloaded and micro-switched pads with force-threshold monitoring and suction cups can be used to place tiles on the floor. A trade-off between excessive compliance to reduce contact forces, and accuracy in placing the tiles needs to be made when designing the contact detection and contact-retention device. Straight or edge-butting placement needs to be experimentally tested to decide on the most feasible approach. Excessive tile bending and compression/tension forces and torques are to be avoided during the installation. Since the required tile placement accuracies are in the sub-millimeter range, and even closed-loop vehicle positioning can only be performed within a few millimeters, an additional sub-millimeter resolution positioner is needed to place the vision system and tile placement device atop the vacant location for the next tile to be properly installed. The currently proposed solution is a limited-travel ( $<1 ")$ XY platform with a full 360 degree rotation table to orient tiles in the center. Vision and tile placement devices ride on the XY-shuttle and rotate with the rotational platform. Tiles are fed to the center of the platform and then translated, oriented, and placed in the proper place on the floor. Such a positioning device can either be forward mounted on the robot, or even underneath the chassis.

Tactile and Compliant Tile Placement Rig: The device used to place tiles should incorporate several features in order to speed up the installation while insuring accurate placement without damaging any tiles. Current designs incorporate linear and rotational compliances (springs), limit switches, and possibly force sensing for control and thresholding. Possible placement scenarios involve straight-line vertical placement or tilted rolling of the tile onto the floor. The placement device will, like the vision system, ride on the limited travel fine-positioning device and have the ability to translate and also rotate the tile to be placed into the proper orientation. Handling of the tiles could be via vacuum feet, or simple grasping and rolling. Further design and experimentation will yield the necessary results to decide on a single solution. [9]

\section{Robot Design and Navigation}

The conceptual design for the tile installation robot is shown in Figure 6. The robot is a four-wheel drive vehicle

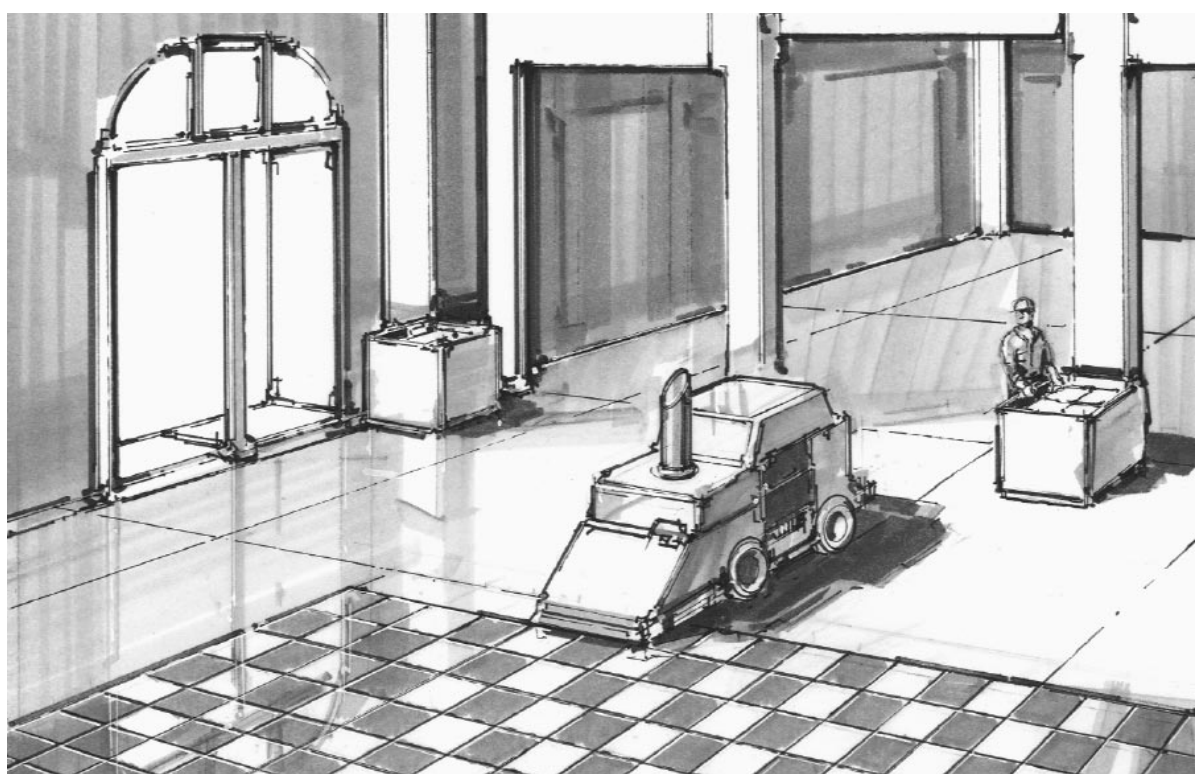

Figure 6: Conceptual Design of a Mobile Robot for Automatic Tile Installation 
with omni-directional wheels. It can emulate various steering modes by controlling the rotational speed and direction of rotation of each wheel. In Figure 7 we have shown the tile hopping and feeding scheme as it feeds into the tile positioning and placement device. Four separate hoppers are serviced by a linear actuator device with vacuum suction cups for handling tiles which are then placed on a conveyor belt. A camera arrangement decides on the quality of the tile itself and trigger the selection device which works similar to those of copier collating devices. If the tile is deemed of good quality the tile is let pass, but otherwise (damage, scuff, wrong pattern) the selector is lowered, and the tile fed into a separate hopper to be retrieved by the operator and put aside for cutting or disposal. Once the tile is forced onto the positioning device via belts, the tile is oriented and displaced in order to be placed onto the ground. The vision system attached to the placement system determines the proper orientation and displacement before the placement. A belt drive combined with a swivelling plate motion and placement device motions will allow an angled placement of the tile.

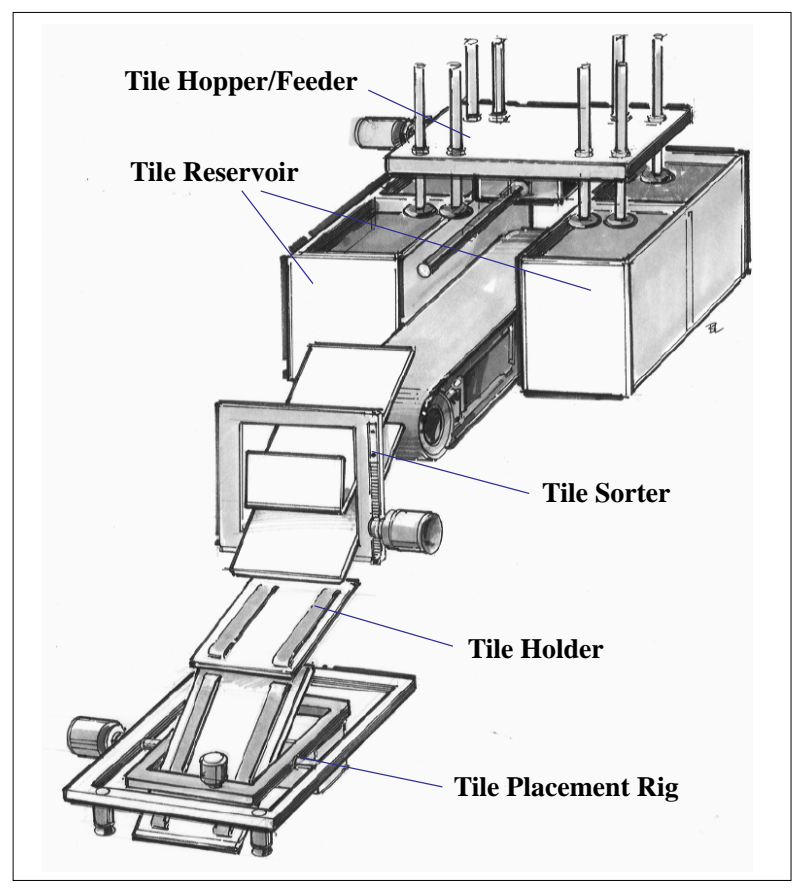

Figure 7: Subsystems for automatic tile installation

Navigation of the robot base within a vacant facility to determine floor plan for future tessellation and placement of the first set of reference tiles, is an important feature of the autonomous robot system. Use of a planar laser-scanning device with retroreflective or active targets would provide for sufficient accuracy to accomplish all the specified tasks. Targets need to only be attached to the facility once at the beginning of the job and allow for accurate navigation over up to 100 meters. Using a planar laser scanner map and navigation from infrared laser reflections off the facility itself is also feasible. Acoustic and infrared proximity sensors provide early warning signals of imminent proximity and collision possibilities and are only used as binary warning sensors, while some of the infrared-proximity sensors can be used to give absolute distance-to-obstacle.

\section{Human/Robot Tiling Performance}

In order to establish a framework for estimating the expected performance of the robot, we have itemized the time-critical non-concurrent sequences which dominate the average time per tile. We contrast this performance with that of a human. This data is based on information from ARMSTRONG World Industries [1], [2], as well as information gathered while on-site for a complete installation process. The robotic performance numbers presented here are estimates based on image processing, computing, and mechanical component performance capabilities.

We observed S\&S Carpet Service tiling up an entirely new K-MART in Ross Park Mall in the North Hills of Pittsburgh. The facility was about $1600^{\prime} \mathrm{x} 45^{\prime}$ and covered $70,000 \mathrm{ft}^{2}$, with the contractor given 3 weeks to finish the job. The following tasks were observed and the time spent on each task was logged, including the tile-laying process. Omitted in the table below, is the time spent measuring out the room, laying and snapping reference chalk-lines, and bringing all the necessary equipment into the facility. Tiles and adhesive supplies had already been placed in the room by the shipping contractor.

\begin{tabular}{|l|l|l|}
\hline \multicolumn{1}{|c|}{ Process } & Specifics & \multicolumn{1}{c|}{ Time } \\
\hline \hline Floor preparation (sweeping, caulking, etc.) & $2,700 \mathrm{ft}^{2}$ & $45 \mathrm{~min}$ \\
\hline Spreading adhesive & $2,700 \mathrm{ft}^{2}$ & $120 \mathrm{~min}$ \\
\hline Loading tiles on dolley & 8 cartons & $15 \mathrm{~min}$ \\
\hline Laying down tiles & 360 tiles & $48 \mathrm{~min}$ \\
\hline
\end{tabular}

Table 2: Human tiling performance

The above table indicates that a human operator takes about 8 seconds to just lay a tile down in the proper place. With all the additional overhead that the operator needs to perform, the average human tiling efficiency lies around $1,200 \mathrm{ft}^{2}$ per shift ( 8 hours) per installer for $12 \mathrm{in}^{2}$ tile, or about 24 seconds per tile. Thus if the overall goal of a robotic system is to reduce the time spent to lay down tiles to about 10 seconds, it could lay around 2,880 tiles in 8 hours. One has to obviously subtract the deployment/ setup/retrieval times from this total deployment time, but even if the robot used 6 hours in a day to purely lay tile, it would lay about 2,150 tiles, which is almost twice the effi- 
ciency of a human operator.

The tile installation robot should spend about 12 seconds for installing a tile and evaluating placement quality. Such performance would lead to a twofold improvement over human installers. The 12 seconds would be spent on the following tasks:

\begin{tabular}{|c|c|}
\hline Process & Time \\
\hline Robot moves to next tile position & $2 \mathrm{sec}$ \\
\hline Acquire \& process images to determine fine position offset & $5 \mathrm{sec}$ \\
\hline Position tile in contact with ground, and lay it down & $3 \mathrm{sec}$ \\
\hline Acquire \& process image to ascertain tiling quality & $2 \mathrm{sec}$ \\
\hline Total Time & $12 \mathrm{sec}$ \\
\hline
\end{tabular}

Table 3: Robot tiling performance

The time spent localizing and checking the tile placement is the dominant component of the overall cycle time. Tile placement will not utilize pure vision to servo the installation, but will rather use vision as initial position offset measures and final installation quality measures. A combination of tactile and force-feedback methods will be employed during tile placement when tiles are edged up and placed next to each other with butting seams. The precision required to move the robot from one tile location to the next has to be within the travel limits of a fine positioning device which has the ability of limited planar translation and rotation to align the next tile to be placed. The error vector could be gathered from the initial image taken of the tiles on the floor, and the edges detected with the light-striping sensors and edge finding algorithms. The accuracy requirement of a tactile/force sensing device to place the tiles from the robot and onto the floor, will be within 4 to 8 thousands of an inch, which will be possible by positioning the tile above the bare floor, and then relying on tactile and force feedback to place the tile with all seams mated properly. Higher bandwidths and more reliable performance can be expected from this scheme. The final step of installation quality assessment will be possible by taking a single image and processing it, to determine the alignment of tile edges.

\section{Summary and Conclusions}

We have configured a four-wheel vehicle with omnidirectional motion capability to automate installation of floor-tiles. The robot utilizes non-contact stereo camerabased sensing to locate tile edges and seams on the floor to place subsequent tiles and to check each placed tile as to quality of installation. A small-motion, high-resolution tile positioning frame carrying the vision and placement sensor and device, is mounted on one end of the vehicle, to achieve the required placement resolution. Coarse robot motions with millimeter accuracy place the sensors within the required vicinity and then allow for accurate fine-positioning. Tiles for the robot are fed from a hooper-system from the opposite end of the vehicle, and supplied via contact-friction belts. The tile handling and placement system is adjustable to different existing and new tile sizes. The use of vinyl and ceramic tiles will be possible using the same hardware setup. In order to check tiling accuracy and track vehicle position and tiling patterns, the vehicle uses a laser-based retroreflective target positioning system.

The automated tile-placement system is expected to exceed the human milestone of 24 seconds spent placing each tile per 8 hour working day. Preliminary cost-benefit analysis indicates that prototype mobile tiling robots should be limited to lay tiles in large commercial acreage areas, leaving the odd and cut tile sections to either human installation, or robot-assisted installation using either specialized tooling or a small special-purpose machine. [9] The robot is to initially be assisted by a human operator for deployment, facility sizing, tiling-job planning and initiation of the tile placement sequence. We envision that the system will eventually be able to measure the facility, accept tiling design and pattern-input from an architect or designer, tessellate out the whole floor, plan the job execution sequence, lay out a reference grid, and place tiles completely on its own. Human intervention will be limited to process monitoring, material feeding, and conflict resolution when the robot encounters an unpredictable condition.

\section{References}

[1] Armstrong World Industries, "Resilient Flooring Installation Manual", 1990.

[2] Armstrong World Industries, "Engineered Installation System", 1991.

[3] Armstrong World Industries, Floor Tile Catalogs.

[4] Ballard, D., and Brown, C., Computer Vision, Prentice-Hall, Inc., Englewood Cliffs, New Jersey, 1982.

[5] Gonzalez, R., and Wintz, P., Digital Image Processing, Addison-Wesley, Reading, MA, 1987.

[6] Mecanum Innovation AB, Sweden, Technical Data, June 1982.

[7] Dowling, K., et al., "A Mobile Robot System for Ground Servicing Operations on the Space Shuttle", Proceedings of Cooperative Intelligent Robots in Space, SPIE OE/Technology, Boston, MA, 1992.

[8] Waldron, K., "The Mechanics of Mobile Robots", Proceedings of the International Conference on Advanced Robotics", pp.533-544, 1985.

[9] Schempf, H., Apostolopoulos, D., "Automated Vinyl and Ceramic Floor-Tile Installation Robot”, Robotics Institute Technical Report, Carnegie Mellon University, to be published. 